\begin{tabular}{lcl}
\hline Bentham OPEN & The Open Biomarkers Journal \\
CrossMark & Content list available at: www.benthamopen.com/TOBIOMJ/ \\
\hline
\end{tabular}

RESEARCH ARTICLE

\title{
Deleted in Malignant Brain Tumor 1 (DMBT1) Expression Pattern in Normal Cervix and at Different Stages of Squamous Intraepithelial Lesions
}

\author{
Andrés Valero ${ }^{1}$, María Lorena Roldán², María Fernanda Ruiz ${ }^{3}$, Juan Manuel Teijeiro ${ }^{1,4}$, Susana \\ Beatriz Marquez ${ }^{3}$ and Patricia Estela Marini ${ }^{1,2,5, *}$ \\ ${ }^{1}$ Laboratorio de Medicina Reproductiva, Facultad de Ciencias Bioquímicas y Farmacéuticas, Universidad Nacional de \\ Rosario, Rosario, Argentina \\ ${ }^{2}$ Instituto de Biología Molecular y Celular de Rosario (IBR-CONICET), Rosario, Argentina \\ ${ }^{3}$ Servicio de Anatomía Patológica, Hospital Provincial del Centenario - Facultad de Ciencias Médicas, Universidad \\ Nacional de Rosario, Rosario, Argentina \\ ${ }^{4}$ Consejo Nacional de Investigaciones Cientificas y Técnicas (CONICET), Rosario, Argentina \\ ${ }^{5}$ Consejo de Investigaciones de la Universidad Nacional de Rosario (CIUNR), Rosario, Argentina
}

Received: May 02, 2017

Revised: August 29, 2017

Accepted: October 30, 2017

\section{Abstract:}

\section{Background:}

Cervical cancer is one of the most frequently occurring malignancies in women worldwide, with high mortality rates. Cervical Squamous Cell Carcinoma (SCC) presents previous states of non-invasive precursor lesions, and early stage Low-Grade Squamous Intraepithelial Lesions (LSIL) regress to normal or Atypical Squamous Cells of Undetermined Significance (ASCUS) in approximately $50 \%$ of cases. Deleted in Malignant Brain Tumors 1 (DMBT1) is a tumour suppression glycoprotein, which absence is considered a malignancy marker in many epithelial cancers.

\section{Objective:}

To analyse DMBT1's presence and localization in SCC and precursor lesions.

\section{Method:}

Immunohistochemistry for DMBT1 was performed in cervix biopsies classified as normal, LSIL, HSIL and SCC.

Results:

DMBT1 was detected at the supranuclear and sometimes infranuclear regions of the endocervix monolayer epithelial cells in normal and HSIL biopsies. In LSIL samples the detection of DMBT1 in endocervix was variable between patients. Also variable was DMBT1 staining in cells of glandular epithelium. The glycoprotein was not detected in the stratified epithelium of the exocervix, regardless of the lesion grade; nor in dysplastic cells.

\section{Conclusion:}

The absence of DMBT1 from endocervix only in some samples of LSIL is promising as a candidate for possible lesion regression potential marker.

\footnotetext{
* Address correspondence to this author at the Laboratorio de Medicina Reproductiva, Facultad de Ciencias Bioquímicas y Farmacéuticas. Universidad Nacional de Rosario. Suipacha 531. S2002LRK. Rosario. Argentina; Tel: 0054-341-4350661; E-mail: pmarini@fbioyf.unr.edu.ar; marini@ibr-conicet.gov.ar
} 
Keywords: DMBT1, Cervix, Cervical Squamous Cell Carcinoma, Squamous Intraepithelial Lesions, Glycoprotein, Epithelium.

\section{INTRODUCTION}

One of the most frequently occurring malignant neoplasms in women worldwide is cervical cancer. It is responsible for high mortality rates and is the fourth most common carcinoma among women on developing countries. Almost $100 \%$ of cervical cancers are related to human papillomavirus (HPV) infections [1]. Such HPV-associated malignancy may be prevented by prophylactic HPV vaccines, which are widely commercially available. However, despite that 58 countries have introduced HPV vaccination in their national immunization programs, one third of girls aged 13-17 have not been fully vaccinated. Also, vaccination programs began in 2003, generally cover only females aged 11 to 13 years old, and the protection vaccines provide is less than $90 \%$ [2]. This means that, although to a much lesser extent, women around the world will continue to develop cervical squamous cell carcinoma (SCC) due to HPV infection.

SCC has well-defined clinical stages, presenting previous states of non-invasive precursor lesions such as Squamous Intraepithelial Lesions (SIL). Morphology remains the standard for diagnosis and characterization of the different cervical lesions [3] and the characteristics of each stage are classified in the thoroughly used Bethesda System Atlas [4]. The full spectrum of carcinogenic lesions that occur in a normal cervix includes Low-Grade SIL (LSIL), High-Grade SIL (HSIL) and carcinoma in situ, whereas tumour progression is composed of locally invasive SCC and distant metastatic lesions. LSIL may regress to normal or atypical squamous cells of undetermined significance (ASCUS) with a frequency of as much of 50\% [5]. Therefore, it is important to identify cases that could potentially develop to HSIL at an early stage of the disease and distinguish them from those with regression potential. Precursor lesions persist longer and progress more quickly in women with oncogenic HPV infections than in women with non-oncogenic infections or without HPV [3]. However, markers other than the presence of certain HPV strains are needed for accurate prognosis of cervical lesions.

Deleted in Malignant Brain Tumors 1 (DMBT1, formerly gp340) is differentially expressed between certain stages of carcinogenesis in many cancer types including colorectal [6], gastric [7], prostate [8], brain [9], breast [10] and lung [11]. This glycoprotein is a member of the Scavenger Receptor Cysteine-Rich (SRCR) family [12] and has been suggested to play important roles in the innate immune defence against pathogens [13, 14], epithelial homeostasis [15], inflammation, and tumour suppression [16]. These functions and their possible mechanisms are under constant study. DMBT1 has been identified in numerous epithelia, bound to membranes, and in secretions such as tears, saliva, bronchoalveolar fluid and pancreatic juice $[13,17]$. In women's genital tract it has been reported in the Fallopian tube, with a suggested role on epithelial protection and homeostasis, possibly related to epithelium-gamete interaction [18]. It has also been studied in cervix, where it is proposed to be an HIV-binding molecule that, on its membrane bound form, facilitates transmission of the virus to CD4+ target cells; in contraposition, its soluble form in fluids would prevent the virus access to the mucosa [19]. In this last work, DMBT1 was detected in cervix by immunohistochemistry in the differentiated spinous layer in the stratified squamous epithelia of the exocervix, with variations between individual samples, and as patches in columnar epithelial cells at the endocervix, ranging from undetectable to intense between individuals [19], however the diverse localization of the glycoprotein was not further analysed.

The presence of DMBT1 in the cervix epithelium, together with the reported variations on its expression during the development of diverse epithelial cancers lead to the hypothesis that DMBT1's expression or localization might vary upon early carcinogenic lesions allowing to distinguish disease progression. To begin testing this hypothesis, in this work we analyse by immunohistochemistry the expression of DMBT1 in cervix biopsies from normal tissue, LSIL, HSIL and SCC.

\section{MATERIALS AND METHODS}

\subsection{Study Design}

A retrospective study was performed on 24 cases of cervical biopsies and conisations performed by Loop Electrosurgical Excision Procedure (LEEP), which were obtained from the archives of Servicio de Anatomía Patológica, Hospital Provincial del Centenario, Facultad de Ciencias Médicas, Universidad Nacional de Rosario (Argentina). The specimens were examined again to corroborate diagnoses under the morphological classification described in the Bethesda System Atlas [4]. The following diagnoses were represented: LSIL (5 cases), HSIL (7 cases), SCC ( 5 cases) and normal ( 7 cases). Patient's age ranged from 21 to 64 years, with a mean and standard deviation for each group of $28 \pm 7,37 \pm 5,52 \pm 7$ and $48 \pm 4$ years old, respectively. Bioethics analysis of the project was precluded 
by Comisión de Bioética, Facultad de Ciencias Bioquímicas y Farmacéuticas, UNR, as samples came from archives. Nevertheless, care was taken to maintain patient's data anonymous. Data of HPV detection by PCR at the moment of treatment were extracted from the clinical history of each patient.

\subsection{Immunohistochemistry Staining}

Histological sections $5 \mu \mathrm{m}$ thick were generated and subsequently applied to silanized slides. Antigen retrieval was performed by microwaving slides for $20 \mathrm{~min}$ in $10 \mathrm{mM}$ trisodium citrate (pH 6). Endogenous peroxidases were inactivated by incubating slides for $30 \mathrm{~min}$ at $4{ }^{\circ} \mathrm{C}$ in $0.3 \% \mathrm{H}_{2} \mathrm{O}_{2}$ in methanol, followed by three 5 -min washes in Trisbuffered saline (TBS, $50 \mathrm{mM}$ Tris-Cl, pH 7.5, $150 \mathrm{mM} \mathrm{NaCl}$ ). Sections were blocked with $5 \%$ skimmed milk, $0.2 \%$ Triton X-100 in TBS for $60 \mathrm{~min}$ and then treated with anti-CUB antibodies (diluted 1:25) overnight at $4^{\circ} \mathrm{C}$. These polyclonal antibodies have been validated for human DMBT1 detection by immunohistochemistry, western blot and immunoprecipitation assays in previous studies $[18,20]$. Secondary antibodies, anti-rabbit IgG-horseradish peroxidase (diluted 1:100), were used in $2 \mathrm{~h}$ incubations. Horseradish peroxidase activity was visualized by development with 3,3'diaminobenzidine tetrahydrochloride and the reaction was stopped by washing with water. Counterstaining was performed by Haematoxylin staining. Finally, the slides were mounted and analysed under Olympus BX40F4 (Japan) microscope. Images were obtained with an Olympus Camedia C60 Zoom camera adapted for microscopy. All phases of the immunohistochemical procedure included samples for positive and negative controls. The positive control was porcine oviduct [20] and for the negative control, preimmune serum was used.

\section{RESULTS}

\subsection{DMBT1 Localization by Immunohistochemistry in Normal Cervix}

Biopsy specimens from normal tissue showed characteristic features for every examined region: endocervix, exocervix and transition zone. DMBT1 signal was detected at the columnar epithelial cells that compose the endocervix, at the supranuclear cytoplasm (Fig. 1A) or at the infranuclear cytoplasm (Fig. 1B) in all of the samples except for one, that didn't present endocervical epithelium (Table 1). DMBT1 was also frequently present in the epithelial cells that surround the glands, at the supranuclear region as well as at the infranuclear cytoplasm (Figs. 1A, C). Signal was undetectable at the stratified squamous epithelium of the exocervix and of the transition zone (Figs. 1D, E). Interestingly, DMBT1 was undetectable in metaplastic cells that invade the DMBT1 positive monolayer epithelia of endocervix and glands (Fig. 1C).

Table 1. DMBT1 detection in endocervix.

\begin{tabular}{|c|c|}
\hline lesion & DMBT1 \\
\hline normal & $+6 / 7$, ni $1 / 7$ \\
\hline LSIL & $+3 / 5,-2 / 5$ \\
\hline HSIL & $+3 / 7$, ni $4 / 7$ \\
\hline
\end{tabular}

+: signal for DMBT1 detected

-: undetectable staining for DMBT1

ni: endocervix region not identified in the biopsy

Table 1 summarizes the variability of DMBT1 detection between patients. As shown, at the endocervical epithelium DMBT1's presence was detected whenever the tissue was conserved in the specimens of patients classified as normal. HPV detection by PCR was negative for all the patients (Table 2).

Table 2. HPV detection in patients with normal and LSIL biopsies.

\begin{tabular}{|c|c|c|c|}
\hline & PATIENT & DMBT1 & HPV \\
\hline \multirow{7}{*}{$\begin{array}{l}\mathbf{N} \\
\mathbf{O} \\
\mathbf{R} \\
\mathbf{M} \\
\mathbf{A} \\
\mathbf{L}\end{array}$} & 1 & + & - \\
\hline & 2 & + & - \\
\hline & 3 & + & - \\
\hline & 4 & + & - \\
\hline & 5 & ni & - \\
\hline & 6 & + & - \\
\hline & 7 & + & - \\
\hline
\end{tabular}




\begin{tabular}{|c|c|c|c|}
\hline & PATIENT & DMBT1 & HPV \\
\hline \multirow{5}{*}{$\begin{array}{l}\mathbf{L} \\
\mathbf{S} \\
\mathbf{I} \\
\mathbf{L}\end{array}$} & $\mathrm{a}$ & + & + \\
\hline & $\mathrm{b}$ & - & + \\
\hline & $\mathrm{c}$ & + & + \\
\hline & $\mathrm{d}$ & - & + \\
\hline & $\mathrm{e}$ & + & - \\
\hline
\end{tabular}

+: signal for DMBT1 detected; positive HPV detection by PCR in serum

-: undetectable staining for DMBT1; negative HPV detection by PCR in serum

ni: endocervix region not identified in the biopsy

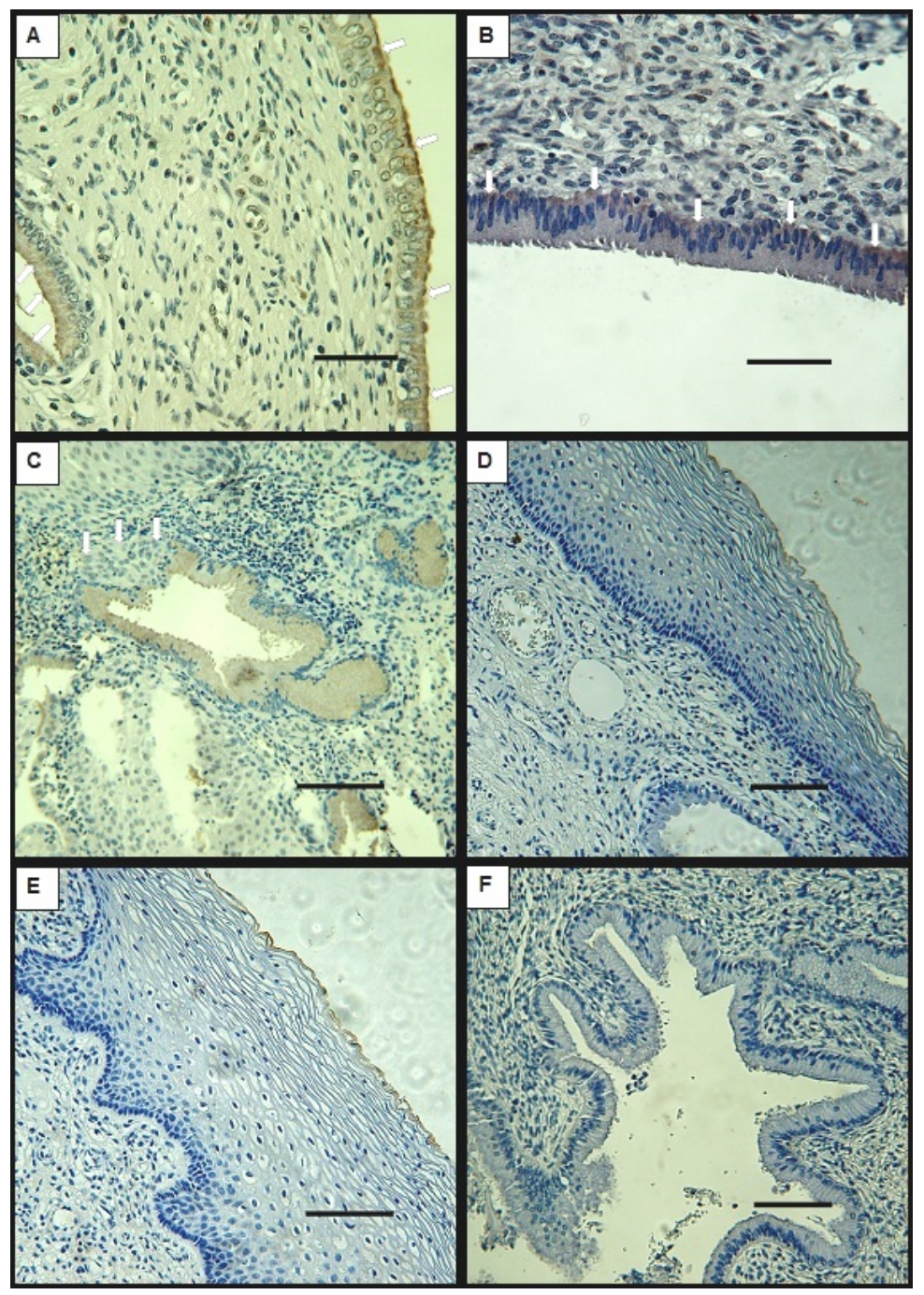

Fig. (1). Representative microphotographs of immunohistochemical analysis for DMBT1 of normal cervix biopsies. A, Bendocervix, arrows show specific staining at supranuclear (A) and infranuclear (B) regions. C- gland positive for DMBT1, arrows show metaplastic cells negative for DMBT1 staining. D- exocervix, E- transition zone, both negative for DMBT1. F- control using pre-immune serum. Bars indicate $50 \mu \mathrm{m}(\mathbf{A}, \mathbf{B})$ and $100 \mu \mathrm{m}(\mathbf{C}, \mathbf{D}, \mathbf{E}, \mathbf{F})$.

\subsection{DMBT1 Localization by Immunohistochemistry in LSIL}

In cervix biopsies classified as LSIL according to the Bethesda System Atlas, DMBT1's signal was detected in some samples that conserved endocervix and glands with normal cell morphology and distribution. In these samples, DMBT1 was present at the supranuclear or infranuclear cytoplasm of the epithelial monolayer cells (Fig. 2C) and at the cells that form some of the glands. However, DMBT1 could not be detected in all of the samples that exhibited normal endocervical tissue (Table 1). As in normal tissue, DMBT1 was undetectable at the stratified epithelia of the exocervix and transition zones (Figs. 2A, B). 


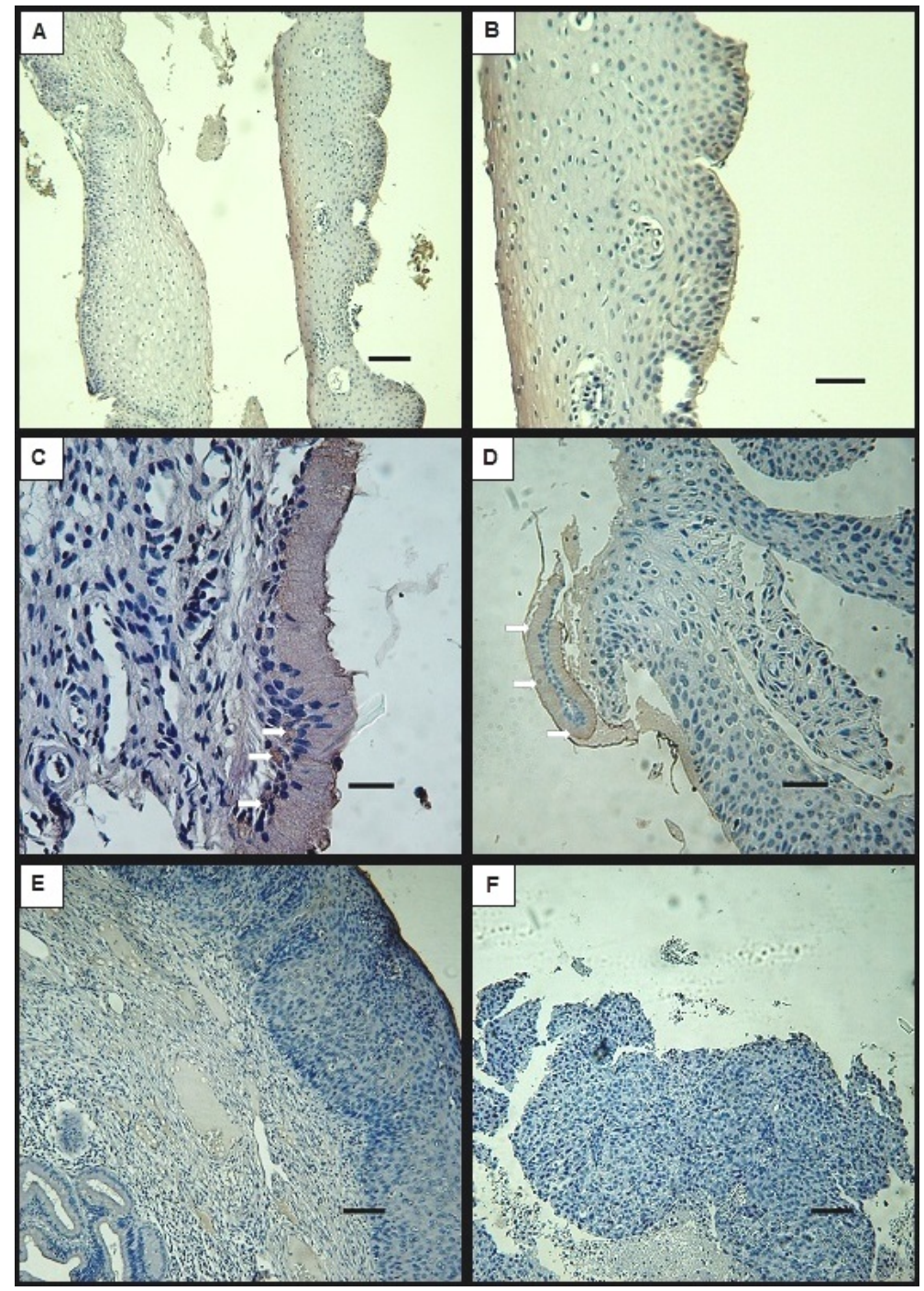

Fig. (2). Representative microphotographs of immunohistochemical analysis for DMBT1 of cervix biopsies with different grades of lesion. A- exocervix from LSIL biopsy, B- 2x amplification of A showing koilocytes. C- endocervix of columnar epithelium with infranuclear DMBT1 specific staining (arrows) in LSIL specimen. D- HSIL specimen showing recognizable detached endocervix monolayer epithelium, positive for DMBT1 staining (arrows). E- exocervix and glands in HSIL specimen. FSCC with non-recognisable cervix regions. Bars indicate $100 \mu \mathrm{m}(\mathrm{A}, \mathrm{E}, \mathrm{F}), 50 \mu \mathrm{m}(\mathrm{B}, \mathrm{D})$ and $25 \mu \mathrm{m}(\mathrm{C})$.

Although the number of examined samples does not allow further analysis, it is to note that the detection of DMBT1 in cervix specimens did not relate to the use of hormonal contraceptive methods. Also, HPV detection by DNA amplification, as registered in the clinical histories, was positive in 4/5 LSIL patients and did not show correlation to DMBT1 detection (Table 2).

\subsection{DMBT1 Localization by Immunohistochemistry in HSIL}

When cervix biopsies classified as HSIL were analysed by immunohistochemistry for DMBT1, the endocervix was absent or not recognizable in most of the samples, but it was positive for DMBT1 in the three samples in which characteristic monolayer epithelia of columnar cells was recognized (Fig. 2D, Table 1). These epithelia were frequently found as detached fragments, devoid of corion (Fig. 2D). Signal was also detected in cells of glandular epithelium in most of the tested samples. In exocervix tissue that conserved integrity, DMBT1 signal was not detected (Fig. 2E). In one of the biopsies the different regions of the cervix could not be recognized. This particular sample showed patches of positive signal (not shown). DMBT1 was not detected in cells showing cytological atypia. 


\subsection{DMBT1 Detection by Immunohistochemistry in SCC}

Cervix biopsies classified as SCC by the Servicio de Anatomía Patológica, Hospital Provincial del Centenario, showed tissue that had lost its integrity preventing the assignment of the typical cervix regions: endocervix, transition zone and exocervix. None of the analysed samples exhibited detectable signal for DMBT1 (Fig. 2F), although minor unspecific staining of some cell debris was noted in some specimens.

\section{DISCUSSION}

The lack or diminution of DMBT1 is considered a malignancy biomarker in many epithelial cancers, and the high regression rate of LSIL promotes the search of biomarkers that allow distinguishing between those lesions that will progress to HSIL and eventually cancer from those that will proceed to regression. However, to our best knowledge, the expression of DMBT1 has not still been analysed in SCC's preceding lesions.

In order to begin studying the role of DMBT1 in cervix cancer establishment, and to challenge the hypothesis that a decrease in this glycoprotein might be a marker of malignancy in the lesions that precede cancer development, we analysed the presence and localization of the glycoprotein in cervix biopsies with different grades of lesion by immunohistochemistry. This technique has become an integral part in diagnostic pathology, as an adjunct method that helps resolve diagnostic dilemmas and can be used as predictive or as prognostic [21].

In cervix, DMBT1 has been assigned an undesirable role in facilitating human HIV binding and transmission to host cells $[19,22]$. It is interesting to note that no difference was observed in DMBT1 localization in HIV positive patients (not shown). Most cervical cancers can be associated to infection by HPV, which causes cervical cancer and its precursor lesions, specifically at the squamocolumnar junction [23, 24], near the transformation zone. Specific cytokine 7 staining patterns have been proposed to distinguish a subpopulation of cells at the squamocolumnar junction which presence indicates potentially aggressive SILs [25]. Although HIV and HPV belong to very different viral types with different mechanisms of infection (lentivirus and Papillomaviridae, respectively), a facilitating effect for DMBT1 in HPV infection, similar to that described for HIV, can be thought. Also, in a transgenic mouse model, the expression of the HPV's E7 oncoprotein in cervix has been shown to produce a decrease of DMBT1's RNA, detected in microarray experiments [26]. This evidence would support a protective role for DMBT1 in cervix, preventing malignancy advance, and the possible use of its diminution as reflection of malignancy progression. However, in this work, no particularity could be assigned to DMBT1's expression in the transition zone in neither of the analysed stages of lesion. In this region, mature squamous cells (negative for DMBT1 staining, Figs. 1 and $\mathbf{2}$ move underneath the endocervical cells, which are positive for DMBT1 in all samples tested, except for some LSIL (Table $\mathbf{1}$ and 2). Also, no correlation was found between HPV infection and DMBT1 expression in human cervix (Table 2).

DMBT1's detection by immunohistochemistry (Table 1) shows that the glycoprotein is detected in endocervix and some gland cells classified as normal by their morphological characteristics in all the normal and HSIL biopsies in which the region could be recognized. In LSIL instead, endocervix devoid of the glycoprotein was observed in some patient's samples. In exocervix, DMBT1 was not detected in any of the analysed specimens disrespectful of the presence or grade of lesion $(\mathrm{n}=17)$. These results are different from the presented by Patyka et al. [19]; who studied biopsies from patients without lesions, finding variations between normal patients, and DMBT1 distributed as patches of granules in few and disperse columnar epithelial cells including the mucosal surface at the endocervix, and as diffuse granular staining throughout the stratified epithelium of exocervix. The difference might respond to the use of different antibodies. While the previous work utilized monoclonal antibodies, in this study we use polyclonal antibodies developed in rabbit and validated by western blot, immunohistochemistry and immunoprecipitation [18, 20], which probably recognize several exposed epitopes of the glycoprotein .

It is to note that DMBT1 was undetectable in cells showing mild or severe alterations in every sample tested from all the analysed stages $(n=17)$. Interestingly, only at the early LSIL stage, when a potent biomarker of malignancy is needed, the expression of DMBT1 in endocervix was variable between samples. Although progression of the illness could not be registered, these results are encouraging as to the possible utility of DMBT1 as a progression marker. Lack of DMBT1 was noted in the biopsies from SCC, but at this stage the integrity of the tissue is completely lost and the histological characteristics are evident.

DMBT1's expression is specifically induced by oestrogen in glandular and luminal epithelia of rat endometrium [27], by progesterone at normal secretory levels in Rhesus monkey endometrium [28], and is greater in ewe cervix secretions in luteal phase than in oestrus [29]. Thus, the possible influence of hormonal contraconception treatment or 
the moment of the female cycle in the expression of DMBT1 in biopsies of human cervix was considered. No relationship was detected, however a greater amount of samples should be analysed to discard ovarian hormonal influences on the cervical expression of DMBT1 in women.

\section{CONCLUSION}

DMBT1 was not detected in altered cells nor in exocervix stratified epithelia, but was present in normal endocervix epithelial cells. Lack of detection of DMBT1 in the endocervical epithelium was exclusive to LSIL grade lesions in precancerous biopsies.

\section{ETHICS APPROVAL AND CONSENT TO PARTICIPATE}

The ethical comitee of facultad de ciencias bioquimicas, univesidad nacional de rosario was consulted. they concluded no human subjects are used, only samples from archives (more than 10 years old), with no possibility of getting in contact with the actual subjects. they considered no ethics evaluation was required. therefore, no file was generated and a number was not assigned.

\section{HUMAN AND ANIMAL RIGHTS}

No Animals/Humans were used for studies that are base of this research.

\section{CONSENT FOR PUBLICATION}

Not applicable.

\section{CONFLICT OF INTEREST}

The authors declare no conflict of interest, financial or otherwise.

\section{ACKNOWLEDGEMENTS}

This work was supported by "Programa de fortalecimiento de las capacidades de investigación y desarrollo" of Provincia de Santa Fe, Argentina (grant number 21010-098-12).

\section{REFERENCES}

[1] Franco EL, Schlecht NF, Saslow D. The epidemiology of cervical cancer. Cancer J 2003; 9(5): 348-59. [http://dx.doi.org/10.1097/00130404-200309000-00004] [PMID: 14690309]

[2] Angioli R, Lopez S, Aloisi A, et al. Ten years of HPV vaccines: State of art and controversies. Crit Rev Oncol Hematol 2016; 102 : 65-72. [http://dx.doi.org/10.1016/j.critrevonc.2016.03.020] [PMID: 27066937]

[3] Chevarie-Davis M, Ramanakumar AV, Ferenczy A, Villa LL, Franco EL. Assessment of the performance of algorithms for cervical cancer screening: Evidence from the Ludwig-McGill cohort study. Gynecol Oncol 2013; 128(3): 415-9. [http://dx.doi.org/10.1016/j.ygyno.2012.12.008] [PMID: 23234804]

[4] Bethesda system for reporting cervical cytology 2014: Online atlas. Available from: https://bethesda.soc.wisc.edu/

[5] Schlecht NF, Platt RW, Duarte-Franco E, et al. Human papillomavirus infection and time to progression and regression of cervical intraepithelial neoplasia. J Natl Cancer Inst 2003; 95(17): 1336-43. [http://dx.doi.org/10.1093/jnci/djg037] [PMID: 12953088]

[6] Peng F, Huang Y, Li MY, et al. Dissecting characteristics and dynamics of differentially expressed proteins during multistage carcinogenesis of human colorectal cancer. World J Gastroenterol 2016; 22(18): 4515-28. [http://dx.doi.org/10.3748/wjg.v22.i18.4515] [PMID: 27182161]

[7] Xiao H, Zhang Y, Kim Y, et al. Differential proteomic analysis of human saliva using tandem mass tags quantification for gastric cancer detection. Sci Rep 2016; 6: 22165. [http://dx.doi.org/10.1038/srep22165] [PMID: 26911362]

[8] Du J, Guan M, Fan J, Jiang H. Loss of DMBT1 expression in human prostate cancer and its correlation with clinical progressive features. urology 2011; 77(2): 509.e9-509.e13.

[http://dx.doi.org/10.1016/j.urology.2010.09.023]

[9] Motomura K, Mittelbronn M, Paulus W, et al. DMBT1 homozygous deletion in diffuse astrocytomas is associated with unfavorable clinical outcome. J Neuropathol Exp Neurol 2012; 71(8): 702-7. [http://dx.doi.org/10.1097/NEN.0b013e31825f2e5d] [PMID: 22805772]

[10] Braidotti P, Nuciforo PG, Mollenhauer J, et al. DMBT1 expression is down-regulated in breast cancer. BMC Cancer 2004; 4: 46-55. [http://dx.doi.org/10.1186/1471-2407-4-46] [PMID: 15301691] 
[11] Srivastava M, Khurana P, Sugadev R. Lung cancer signature biomarkers: Tissue specific semantic similarity based clustering of digital differential display (DDD) data. BMC Res Notes 2012; 5: 617. [http://dx.doi.org/10.1186/1756-0500-5-617] [PMID: 23122428]

[12] Aderem A, Ulevitch RJ. Toll-like receptors in the induction of the innate immune response. Nature 2000; $406(6797): 782-7$. [http://dx.doi.org/10.1038/35021228] [PMID: 10963608]

[13] Madsen J, Mollenhauer J, Holmskov U. Review: Gp-340/DMBT1 in mucosal innate immunity. Innate Immun 2010; $16(3)$ : $160-7$. [http://dx.doi.org/10.1177/1753425910368447] [PMID: 20418254]

[14] Reichhardt MP, Meri S. SALSA: A regulator of the early steps of complement activation on mucosal surfaces. Front Immunol 2016; 7: 85. [http://dx.doi.org/10.3389/fimmu.2016.00085] [PMID: 27014265]

[15] Kang W, Reid KB. DMBT1, a regulator of mucosal homeostasis through the linking of mucosal defense and regeneration? FEBS Lett 2003; 540(1-3): 21-5.

[http://dx.doi.org/10.1016/S0014-5793(03)00217-5] [PMID: 12681477]

[16] Ligtenberg AJ, Veerman EC, Nieuw Amerongen AV, Mollenhauer J. Salivary agglutinin/glycoprotein-340/DMBT1: A single molecule with variable composition and with different functions in infection, inflammation and cancer. Biol Chem 2007; 388(12): 1275-89. [http://dx.doi.org/10.1515/BC.2007.158] [PMID: 18020944]

[17] Malamud D, Wahl SM. The mouth: A gateway or a trap for HIV? AIDS 2010; 24(1): 5-16. [http://dx.doi.org/10.1097/QAD.0b013e328333525f] [PMID: 19935380]

[18] Roldán ML, Marini PE. First evidence of the interaction between deleted in malignant brain tumor 1 and galectin-3 in the mammalian oviduct. Histochem Cell Biol 2014; 141(2): 181-90. [http://dx.doi.org/10.1007/s00418-013-1145-2] [PMID: 24065275]

[19] Patyka M, Malamud D, Weissman D, Abrams WR, Kurago Z. Periluminal distribution of HIV-Binding target cells and Gp340 in the Oral, cervical and sigmoid/rectal mucosae: A mapping study. PLoS One 2015; 10(7): e0132942. [http://dx.doi.org/10.1371/journal.pone.0132942] [PMID: 26172445]

[20] Teijeiro JM, Roldán ML, Marini PE. Molecular identification of the sperm selection involved porcine Sperm Binding Glycoprotein (SBG) as Deleted in Malignant Brain Tumors 1 (DMBT1). Biochimie 2012; 94(1): 263-7. [http://dx.doi.org/10.1016/j.biochi.2011.10.008] [PMID: 22051378]

[21] Desouki MM, Chamberlain BK, Li Z. The role of immunohistochemistry in the evaluation of gynecologic pathology part 2: A comparative study between two academic institutes. Ann Diagn Pathol 2015; 19(5): 296-300. [http://dx.doi.org/10.1016/j.anndiagpath.2015.06.005] [PMID: 26141018]

[22] Stoddard E, Cannon G, Ni H, et al. gp340 expressed on human genital epithelia binds HIV-1 envelope protein and facilitates viral transmission. J Immunol 2007; 179(5): 3126-32. [REMOVED HYPERLINK FIELD]. [http://dx.doi.org/10.4049/jimmunol.179.5.3126] [PMID: 17709527]

[23] Bosch FX, Lorincz A, Muñoz N, Meijer CJ, Shah KV. The causal relation between human papillomavirus and cervical cancer. J Clin Pathol 2002; 55(4): 244-65. [http://dx.doi.org/10.1136/jcp.55.4.244] [PMID: 11919208]

[24] Marsh M. Original site of cervical carcinoma; topographical relationship of carcinoma of the cervix to the external os and to the squamocolumnar junction. Obstet Gynecol 1956; 7(4): 444-52. [PMID: 13309917]

[25] Mills AM, Paquette C, Terzic T, Castle PE, Stoler MH. CK7 Immunohistochemistry as a Predictor of CIN1 Progression: A Retrospective Study of Patients From the Quadrivalent HPV Vaccine Trials. Am J Surg Pathol 2017; 41(2): 143-52. [http://dx.doi.org/10.1097/PAS.0000000000000747] [PMID: 27680604]

[26] Sierra EI, Chávez JD, Cortés-Malagón EM, et al. Differential gene expression between skin and cervix induced by the E7 oncoprotein in a transgenic mouse model. Virology 2012; 433(2): 337-45. [http://dx.doi.org/10.1016/j.virol.2012.08.024] [PMID: 22980503]

[27] Tynan S, Pacia E, Haynes-Johnson D, et al. The putative tumor suppressor deleted in malignant brain tumors 1 is an estrogen-regulated gene in rodent and primate endometrial epithelium. Endocrinology 2005; 146(3): 1066-73. [http://dx.doi.org/10.1210/en.2004-1304] [PMID: 15564322]

[28] Ace CI, Okulicz WC. A progesterone-induced endometrial homolog of a new candidate tumor suppressor, DMBT1. J Clin Endocrinol Metab 1998; 83(10): 3569-73. [PMID: 9768666]

[29] Soleilhavoup C, Riou C, Tsikis G, et al. Proteomes of the female genital tract during the oestrous cycle. Mol Cell Proteomics 2016; 15(1): 93-108. [http://dx.doi.org/10.1074/mcp.M115.052332] [PMID: 26518761]

This is an open access article distributed under the terms of the Creative Commons Attribution 4.0 International Public License (CC-BY 4.0), a copy of which is available at: (https:/creativecommons.org/licenses/by/4.0/legalcode). This license permits unrestricted use, distribution, and reproduction in any medium, provided the original author and source are credited. 